\title{
Tuberkulose im althistorischen Hellas
}

\author{
Tuberculosis in the Old Medical History of Greek
}

\section{Einleitung}

In den frühesten Heilbehandlungen in Griechenland spiegeln sich Elemente aus Magie, Mythologie und religiösem Götterkult wider. Apollo, in der Sage Vater des Asklepios, sowie Athena, Artemis, Eileithya und viele weitere Gottheiten bilden dominierende Elemente in der griechischen Mythologie der Heilkunst, in der die Grenzen zwischen dem Gott als Arzt und dem Arzt als Gott fließend und retrospektiv nur mit Einschränkungen zu definieren sind. Ohne Zweifel wurden durch die Mysterien und religiösen Riten, die auch Formen des Exorzismus und Opferkult umfassten und damit Analogien zur assyrisch-babylonischen Praxis zeigten, psychotherapeutische Effekte ausgelöst. Neben diesen rituellen Phänomenen entwickelte sich aber auch früh ein praktisches Sachwissen über die Wirkung von Heilpflanzen und die Möglichkeiten der Wundchirurgie. Wie aus mündlichen Überlieferungen bekannt, besaßen z. B. Chiron als Zentaur aus Thessalien um 1270 v. Chr. oder seine Schüler Asklepios (um 1260 v. Chr. in Thessalien geboren) und Aristaios (Geburtsort Kyreneika, Libyen) umfangreiche heilkundliche Erfahrungen, die sich nicht nur auf die Versorgung von Verletzungen beschränkten, sondern sich auch auf Symptome innerer Erkrankungen bezogen [1]. Über Asklepios, der einerseits als Heil- und Arztgott der Antike [2], andererseits als thessalischer Heros und Arzt bezeichnet wird [3], existieren zahlreiche Legenden, die sich mit esoterischen Wunderheilungen, Epiphanie und Totenerweckungen befassen. Es sind allerdings - ebenso wie aus der Epoche der minoischen Kultur zwischen 2000-1400 v. Chr. - nur wenige schriftliche Dokumente erhalten.

\section{Althistorische hellenistische Schriftdokumente - Quellenlage zur Tuberkulose}

\section{Älteste Schriften - Ilias und Odyssee}

$\mathrm{Zu}$ den ältesten erhaltenen griechischen Schriften gehören die Werke Ilias und Odyssee [4], in denen früheste Vorstellungen von Krankheit und Verletzungen als Folge kriegerischer Auseinandersetzungen zum Ausdruck kommen [5]. Beide Epen über den trojanischen Krieg und die späte Heimkehr des Odysseus wurden nach heutigem Wissenstand von Homer verfasst, obgleich im Vergleich beider Dichtungen zahlreiche disparate Inhalte zu finden sind, die an einem alleinigen Autoren zweifeln lassen [6]. Homer (Abb. 1) stammte historischen Recherchen zufolge aus der Küstenstadt Smyrna/Izmir und lebte vermutlich im 8. Jahrhundert v. Chr. [7]. Basierend auf epischen Traditionen finden sich in der Ilias auch Lieder, Heldensagen und Kleinepen wieder, die zuvor lediglich mündlich über Generationen aus der Zeit der frühgriechischen Stämme überliefert wurden, welche zwischen 1100 und 900 v. Chr. unter der Führung verschiedener Könige in den Mittelmeerraum vordrangen. Ob und in welchem Umfang sich in der Ilias Fragmente der kretisch- mykenischen Kultur der frühmykenischen Periode (1500 - 1425 v. Chr., [8]) widerspiegeln und damit noch deutlich ältere Elemente enthalten sind, ist nach der versuchten Entzifferung der Linear-B-Schrift Thema kontroverser Diskussionen [7].

Im homerischen Arzttum behandeln die beiden Protagonisten Machaon und Podaleirios im Rahmen der Kriegschirurgie ausschließlich Traumata [9]. Erst in der nachhomerischen Tradition

Rubrikherausgeber: R. Kropp (federführend), U. Costabel, H. S. Fuchs, C. Habrich, H. Jungbluth, H. J. Klippe, N. Konietzko, R. Loddenkemper, G. Neumann, S. Schulz, M. Teschner

Institutsangaben Klinik für Thoraxchirurgie, Klinikum Delmenhorst 


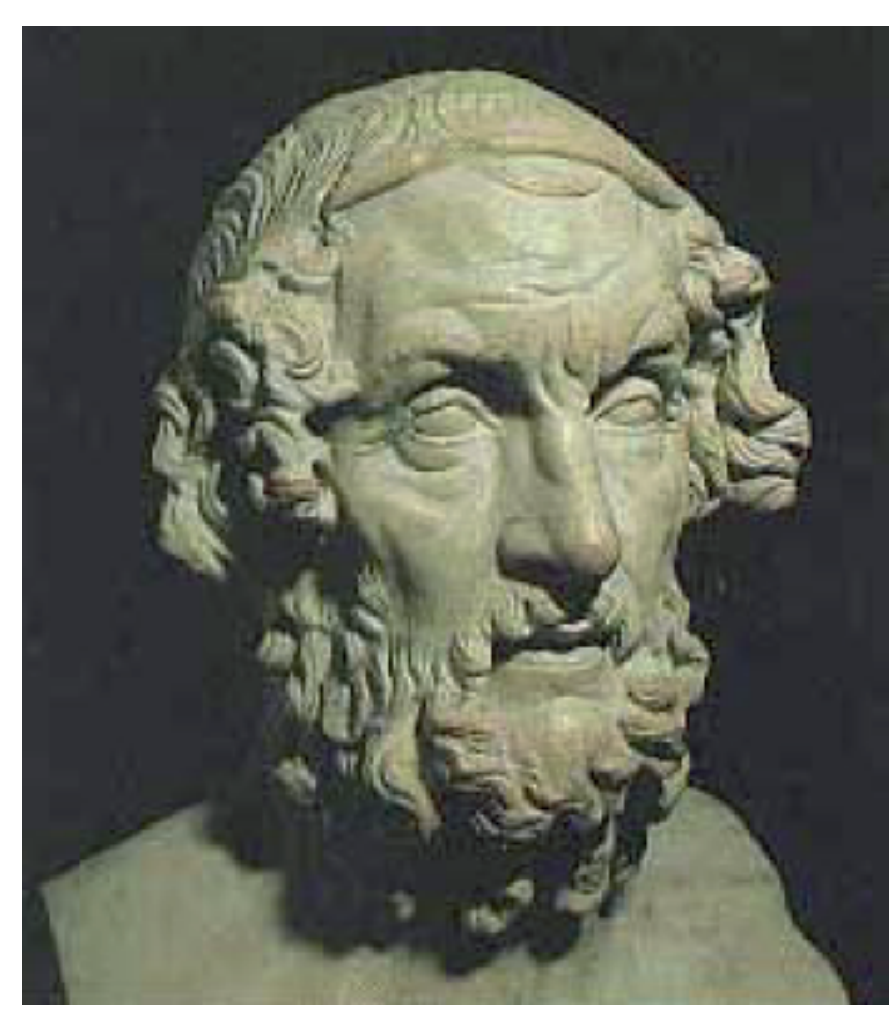

Abb. 1 Homer

erscheint Podaleirios als Begründer der Internistik [10], wie entsprechende Verse im „Iliupersis“ des kyklischen Epikers Arktinos belegen [11]. Dieses Werk entstand vermutlich im 7. Jahrhundert v. Chr. In den homerischen epischen Dichtungen gelten nichttraumatisch bedingte Krankheiten als nicht zu erkennen und für unheilbar.

Dementsprechend finden sich weder in der Ilias noch in der Odyssee heilkundliche Angaben zur Behandlung innerer Erkrankungen, zumal diese Krankheiten im Gegensatz zu den empirisch-rational begriffenen Wunden als Strafe eines Gottes oder eines Dämons angesehen wurden. Der Erkrankte wurde als Sünder gesellschaftlich stigmatisiert $[12,13]$. Gleiches galt auch für den Verlauf langwieriger Verletzungsfolgen: Die chronische Krankheit resultierte nach Auffassung der epischen Dichter aus einer göttlichen Reaktion (zum Beispiel als Strafe bei der Verletzung eines Heiligtums), wobei das langdauernde „Dahinschwinden" als Makel empfunden wurde, dem der rasche heldenhafte Tod im Kampf immer vorzuziehen war. Über das „Dahinsiechen“ ist im 2. Gesang der Ilias (II, Verse 721 - 725) vermerkt (Übersetzung von J. H. Voss, 2000 [14]):

„Jetzt aber lag er (Philoktetes, Anm. d. Verfassers), von Qualen verzehrt, auf der heiligen Insel Lemnos, wo ihn das Volk der Achaier hatte verlassen, Siech vom tödlichen Biß der unheilspeienden $\mathrm{Hy}$ dra. Jammernd lag er daselbst; allein die Männer von Argos sollten sich bald bei den Schiffen des Herrn Philoktetes erinnern."

Sophokles aus dem attischen Demos Kolonos (497/96 - 406/05 v. Chr. [15]) formuliert in seiner Tragödie „Philoktetes“, die $409 \mathrm{v}$. Chr. im Dionysostheater Athen aufgeführt wurde [16], dass der verletzte Heerführer ausgesetzt und seinem Schicksal überlassen wurde ([1]. Epeisodion, V). Der Krankheitsverlauf $\varepsilon \varsigma \varphi \theta i ́ \sigma v$ (phthimo) wird mit hinschwinden, umkommen, vergehen übersetzt und verdeutlicht den langdauernden progredienten Kräfteverfall. F. Kudlien übersetzt den Verlauf mit „der an der schlimmen Krankheit Hinschwindende" als chronischen Prozess [17], W. Willige übersetzt (Ph $254-9,1966$ [18], S. 564):

„Sie aber, die mich frevelhaft hier ausgesetzt, die lachen insgeheim, indessen mein Geschwür gedeiht und fressend immer weiter um sich greift."

Ähnliche Formulierungen finden sich in der Odyssee, wo der Kranke ebenfalls „lange dahinschwindet“ [17]. Die Termini bezeichnen den Kräfteverlust im Rahmen des chronischen Krankheitsverlaufs, nicht jedoch die Schwindsucht als Synonym für die Tuberkulose, für die sich bei Homer keine klinischen Hinweise finden lassen.

Neben der Ilias und der Odyssee existieren mit den Epen Kyklos, Kypria, Margites und weiteren homerischen Hymnen zahlreiche Dichtungen, deren Redaktion ebenfalls in die Epoche der frühesten griechischen Schriften fällt. In den Überlieferungen der Asklepiaden aus der Zeit um $800 \mathrm{v}$. Chr. finden sich keine Hinweise auf die Tuberkulose.

\section{Herodot und die Urhistorie des Begriffs „Phthisis“}

Beeinflusst durch die mathematischen und an den Gesetzen der Natur orientierten Vorstellungen von Pythagoras von Samos (um $582-480$ v. Chr.) versuchten Vorsokratiker wie Thales von Milet (624-548 v. Chr.), Anaximenes von Milet (570-548 v. Chr.) und Heraklit von Ephesos (556 - 548 v. Chr.), medizinische Phänomene weniger durch mythisch und magisch-religiöse Erklärungen zu begreifen, sondern auf rationaler Ebene zu verstehen [22,23]. Von Herodot aus Halikarnossos im Bodrum (Kleinasien; Lebenszeit ca. 485/4 - 430/25 v. Chr.; Abb. 2) ist das unvollendete, später in 9 Bücher geteilte Werk erhalten, das die historischen Ereignisse vom Trojanischen Krieg bis zu Xerxes' Zug gegen Griechenland mit der Schlacht bei Mykale $479 \mathrm{v}$. Chr. umfasst [24,25]. In diesen Schriften sind ausführliche ethnografische Exkurse über die Lyder, Perser, Babylonier und Massageten sowie die Skythenund Libyenfeldzüge der Perser enthalten [26,27]. Hinweise auf klinische Symptome oder Beschwerden, die auf eine spezifische Erkrankung deuten, finden sich nicht.

Aus Herodots erhaltenen Schriften sind Hinweise auf Heilpflanzen aus Arabien und Rausch- und Suchtmittel bei den Skythen zu finden. Die Beschreibung von Symptomen erlaubt die Identifikation von Haut- und venerischen Krankheiten (z. B. die Krätze) sowie von einigen Infektionskrankheiten (zum Beispiel der Pest [28]). Die Ausführungen erfolgen aus situativ-historischer, jedoch nicht aus medizinischer Sicht [29], so dass nur wenige klinische Angaben zu einzelnen Krankheitssymptomen notiert sind. Ausführlicher werden verschiedene Bestattungsriten und Formen von Techniken zur Leichenbalsamierung der Ägypter, Perser, Babylonier und Skythen dargestellt [30].

Es sind keine klinischen Symptome oder Krankheitsprozesse zu recherchieren, die auf die Existenz der Tuberkulose schließen lassen. Im Zusammenhang mit der Verletzung eines Kriegsführers wird jedoch der Terminus $\varphi \theta i$ í $\varepsilon^{\prime} v o r$ (phthimenoi) verwendet, 


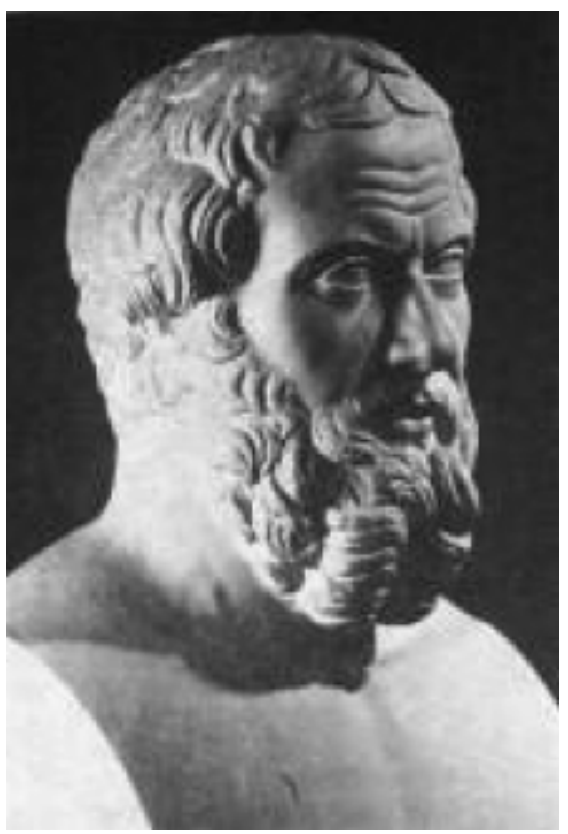

Abb. 2 Herodot

übersetzt mit die Dahinsiechenden oder die Toten (Buch III, Übersetzung von F. Lange, 1885 [31], S. 158):

„Oberste der Reiterei (Anm.: im Zuge des Xerxes gegen Hellas) waren Armamithres und Tithäos, Söhne des Datis. Der dritte Oberste der Reiterei, Pharnuches, war in Sardes krank zurückgeblieben. Nämlich wie sie aufbrachen von Sardes, begegnete ihm ein unerwartetes Unglück. Als er nämlich so ritt, lief ein Hund seinem Pferd unter die Beine, und das Pferd sah nicht vor sich, scheute, bäumte sich auf und warf den Pharnuches ab. Und er spie Blut nach dem Fall, und die Krankheit artete in Schwindsucht aus."

In der Übersetzung von F. Kudlien (1967 [32], S. 110) heißt es:

„... die Krankheit ging in ein Dahinschwinden (...) über.“

In der Übersetzung von Horneffer ([33], VII Buch, Xerxes 486 - 465, Heer und Flotte der Perser, 1955):

„Reiterführer waren Harmamithres und Tithaios, Söhne des Datis. Der dritte Reiterführer, Pharnuches, war krank in Sardes zurückgeblieben. Beim Ausmarsch aus Sardes hatte sich ein widriger Unglücksfall ereignet. Ein Hund lief zwischen die Füße seines Pferdes, das erschreckt sich bäumte und Pharnuches abwarf. Er fiel und spie Blut, und schließlich ging die Krankheit in Schwindsucht über.“

Es handelt sich um die früheste altgriechische Textquelle, in der sich der Terminus $\varphi \theta i ́ \sigma \varsigma \varsigma$ (phthisis) findet [34]; im genuinen Kontext wird der Krankheitsverlauf nach einer Verletzung beschrieben. Für eine Tuberkulose oder eine durch das Trauma begünstigte Exazerbation eines alten spezifischen Infektionsherdes gibt es keine klinischen Hinweise.

\section{Corpus Hippocraticum}

Sowohl aus der archaischen Epoche mit ihren Vorstufen zwischen 800 - 500 v. Chr. [35,36], als auch aus dem Beginn der klassischen Epoche um 500-400 v. Chr. sind keine Schriftsammlun- gen erhalten, die ausschließlich heilkundliche Themen behandeln.

Als frühestes systematisches medizinisches Werk gilt das Corpus Hippocraticum, einer Sammlung aus ca. sechzig Schriften, die von Hippokrates aus Kos als Begründer der rational-empirischen Medizin (um 460 - 377 v. Chr.) und von verschiedenen weiteren unbekannten Autoren während der hellenistischen Epoche zwischen 450-350 v. Chr. verfasst wurden (Abb. 3) [37]. Die hippo-

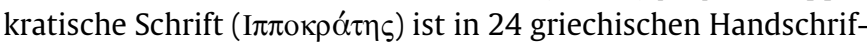
ten nahezu vollständig erhalten [38].

Lediglich das Kapitel „Das Sehen“ aus der Schrift „Die inneren Krankheiten" ist nur inkomplett vorhanden [39]. Das Corpus Hippocraticum umfasst Texte mit allgemeinen heilkundlichen Vorschriften, Krankheitsberichten, Diagnosen, Prognosen, Beschreibungen einzelner Krankheiten und Diätanweisungen, geprägt von den Vorstellungen der sog. Humoralpathologie, die davon ausgeht, dass Krankheiten bei einem Missverhältnis und bei ungenügender Mischung der Säfte entstehen [40].

\section{Hippokrates und die Tuberkulose}

Bei der Übersetzung des Corpus Hippocraticum wird der Terminus phthoë synonym für phthisis $\varphi \theta i ́ \sigma ı \varsigma$ verwendet [41]. Phthisis und phthoë finden vorwiegend in den Schriften der Vorhersagungen (Prognostikon) Erwähnung sowie im 1. und 2. Buch der inneren Krankheiten im Zusammenhang mit der Verbreitung, vermuteter Ätiologie und Pathogenese, Prognose, klinischer Symptomatik und Behandlung der Erkrankung [42]. Beide Termini werden im deutschsprachigen Raum in der Regel als Schwindsucht oder Phthisis [42,43], im angloamerikanischen Raum als consumption und im französischen Sprachgebrauch als phthisie [44] übersetzt.

In den Schriften der Volkskrankheiten (3. Buch [45]) wird zur Verbreitung der als Schwindsucht übersetzten Erkrankung gesagt (Lehrsatz 13, Kapitel „Seuchenhafter Jahrgang“ [45], S. 92):

„Als das größte und schwerste Leiden, welches die meisten tötete, herrschte die Schwindsucht. Viele ihr Anheimfallende hatten schon den Winter über zu kränkeln begonnen, wovon ein großer Teil darniederlag; die anderen ertrugen ihre Leiden aufrecht. Von den Bettlägerigen starben die meisten mit Frühlingsbeginn; bei keinem einzigen der anderen setzte der Husten aus, milderte sich indessen den Sommer über; im Laufe des Herbstes aber kamen alle zum Liegen und viele starben. Die meisten von ihnen siechten lange dahin; bei der Mehrzahl von diesen begann die Verschlimmerung ganz plötzlich; es kamen gehäufte Frostschauer, oft anhaltende heftige Fieber, reichliche unzeitige kalte Schweiße bis zum Ende; die Kranken kühlten dabei ab und konnten kaum wieder erwarmen. ... der Husten blieb immerfort bis zum Ende häufig und führte viel gekochten und flüssigen Auswurf nicht allzu mühsam hervor. ... Das bei weitem größte Unheil entstand für diese Kranken wie für die anderem aus dem Widerwillen gegen Speisen, wie schon erwähnt wurde. ... Unter Frösteln und Irrereden starben sie.“

Im Prognostikon finden sich im Kapitel der koischen Vorhersehungen 640 Lehrsätze, die klinische Kriterien zum Erkrankungsverlauf vermitteln. 153 Sätze wurden aus den 170 Lehrsätzen des 


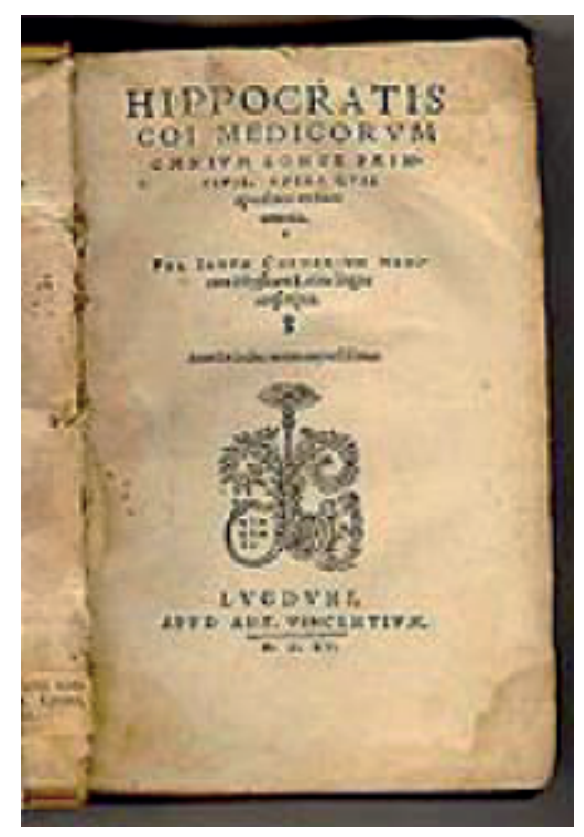

Abb. 3 Corpus hippocraticum

Prorrheticons übernommen [45]. Über die „Schwindsucht“ finden sich nachfolgende Hinweise ([45], S. 38):

„Neigung zu Fieberfrösten und Atemnot bei körperlicher Anstrengung sind Vorzeichen von Schwindsucht.“

Teil 13, 254 ([45], S. 61):

„Häufige Anfälle von Sprachlosigkeit in totenschlafähnlichem Zustande künden eine zukünftige Neigung zur Schwindsucht an.“

Teil 13, 295 (Koa. 281, 434 [45], S. 67):

„Wenn unter Stockung des Gedärms die Unterrippengegenden sich erheben, so steht es schlimm: am schlimmsten steht es unter den langwierig Kranken für die Schwindsüchtigen und für andere, die an flüssiges Gedärm gewöhnt sind.“

Teil 13, 398 (Aph. 208 [45], S. 79):

„Bei denen in der Brust Entzündungsherde (phyma) entstehen, die husten den Eiter aus bis zu vierzig Tagen nach dem Durchbruch; wenn sie diese Zeit überstanden haben, werden sie fast ausnahmslos schwindsüchtig.“

Teil 13, 430 und 436 ([45], S. 83):

„Die Schwindsucht ist am gefährlichsten, die mit der Zerreißung großer Blutadern beginnt und mit einem Schleimfluß vom Kopf her. Atemnot ohne Auswurf aber mit reichlichem Aushusten von unverdautem Auswurf ist in der Schwindsucht verderblich.“

Im Zusammenhang mit der vermuteten Pathogenese der entzündlichen Geschwüre in der Lunge (XVII/ 64 [45], S. 64):
„Ursachen: Galle- oder Schleimansammlung - Fauligwerden derselben - Höhlenbildung.

Prognose:

- bei Eiterentleerung und Austrocknung der Höhle: Gesundung.

- Bei ausbleibender Austrocknung: Durchfall, Tod,

- Bei fehlender Eiterentleerung: Allmähliches Schwächerwerden, Tod,

- Bei Eiterdurchbruch nahe dem Zwerchfell: Gesundung,

- Bei langem Verweilen des Eiters aber ebenfalls Tod.“

Im 2. Abschnitt der hippokratischen Lehrsätze finden sich Hinweise über den Einfluss der Jahreszeiten auf Schwindsüchtige ([45], XIV, S.37), die Häufigkeit der Schwindsucht ([45], XIV, S. 39) und die Altersverteilung ([45], XIV, S. 79):

„Bei jungen Leuten gibt es Blutspeien, Schwindsucht, hitzige Fieber, Fallsucht und andere Krankheiten; am meisten aber eben die genannten. Schwindsucht (phthisis) entsteht zumeist auf der Alterstufe zwischen dem achtzehnten und fünfunddreißigsten Lebensjahr.“

Das 1. Buch der Krankheiten, möglicherweise von Galen und Dioskurides von Thessalos oder Polybos verfasst, ggf. aber auch von knidischer Herkunft, behandelt in zwei größeren Abschnitten verschiedene Arten von Empyemen und die Ätiologie und Pathogenese der so genannten akuten Krankheiten. In den Kapiteln 48-65 des 2. Buches werden die Symptome von verschiedenen Lungenerkrankungen und die Möglichkeiten der Behandlung erläutert. Über die Symptomatik und Therapie der phthisis ist dokumentiert ([45], XVIII, S. 62):

„Es befällt den Kranken Husten, der Auswurf ist reichlich und flüssig und wird bisweilen leicht ausgehustet, der Eiter ist wie Hagelkörner und wenn man ihn zwischen den Fingern zerreibt, ist er hart und übelriechend. Die Stimme ist rein, und das Sprechen verursacht keinen Schmerz, Fieber sucht ihn nicht heim, wohl aber bisweilen Hitze, im Übrigen isst er auch schwach.

Einem solchen muss man Nieswurz und Linsenwasser zu trinken und soviel als möglich zu essen geben, wobei er sich scharfer Speisen, des Rindfleisches, Schweinefleisches und Schaffleisches enthalten soll. Er mache nur wenig Gymnastik, er gehe spazieren, nach dem Essen erbreche er. Er enthalte sich geschlechtlicher Aufregungen. Diese Krankheit währt sieben oder neun Jahre.“

In der Schrift der Inneren Krankheiten des Corpus Hippocraticum ([45], XIX) kommen die Vorstellungen zur Ätiologie, Pathogenese und Therapie in der hippokratischen Zeit ausführlich zur Darstellung, geprägt von den Lehren der knidischen Ärzteschule. Bei der Schwindsucht werden drei Formen unterschieden ([45], X. S. 36):

„Es gibt drei Arten von Schwindsucht. Die erste Art. Diese entsteht durch Schleim. Wenn die mit Schleim angefüllte Kopfhöhle erkrankt und (in ihr) Hitze austritt, so verfault der Schleim in der Kopfhöhle, weil er sich nicht soweit bewegen kann, dass er abgeht. Wenn er sich dann verdichtet hat und verfault ist und die Äderchen übervoll (von ihm) geworden sind, dann entsteht ein Fluß nach der Lunge. Wenn die Lunge ihn in sich aufgenommen hat, erkrankt sie 
sogleich, weil sie von dem Schleim, der salzig und faulig ist, angegriffen wird.

Der Kranke hat nun folgendes zu leiden. Es befällt ihn anfangs ein gelindes Fieber und Frost, er hat Schmerz an der Brust und am Rücken, zuweilen quält ihn auch heftiger Husten, und er gibt reichlich flüssigen und salzigen Auswurf von sich. Dies hat er bei Beginn der Krankheit zu leiden. Im weiteren Verlauf derselben aber magert der Körper ab, die Schenkel ausgenommen, diese schwellen vielmehr an, die Füße desgleichen, und die Nägel verziehen sich. An den Schultern wird er mager und kraftlos, der Schlund füllt sich mit einer Art Schaum und pfeift wie durch ein Rohr. Während der ganzen Krankheit hat man heftigen Durst, und den Körper befällt eine hochgradige Schwäche. Ein solcher geht unter diesen Umständen in einem Jahr elend zugrunde und stirbt.

Die zweite Art der Schwindsucht. Sie entsteht durch übermäßige Anstrengung. (Der Kranke) hat meistenteils dasselbe durchzumachen wie der Vorbeschriebene. Diese Krankheit hat aber mehr Unterbrechungen als die vorige und lässt im Sommer nach. ... sowie er sich ein wenig anstrengt, befällt ihn (Lungen-)Blähung und Atemnot. Ein solcher stirbt an dieser Krankheit in der Regel innerhalb von drei Jahren.

Die dritte Art von Schwindsucht. Infolge dieser hat (der Kranke) folgendes durchzumachen. Sein Rückenmark wird voll Blut und Galle. Er zehrt aber gleicherweise auch von den hohlen Adern her ab, diese füllen sich mit wässrigem Schleim und mit Galle. Sie haben das gleiche durchzumachen, von welcher dieser beiden (Veränderungen) die Schwindsucht kommt. ... Die Krankheit währt gewöhnlich neun Jahre, hierauf geht er zugrunde. Wenige entrinnen ihr, denn die Krankheit ist schwer."

Im Abschnitt über „Die Flüssigkeiten und ihre Anwendung“ werden anatomisch-physiologische Vorstellungen deutlich und Hinweise auf Komplikationen entzündlicher Lungenprozesse und deren Behandlung gegeben (Kap. 14 - 18 [45], VIII, S. 64):

„Nach der Lunge geht der Fluß (Anm.: der Säftefluss) beständig in geringer Stärke und bewirkt keine große Durchfeuchtung in der Lunge. Das Hinzuströmende wird nämlich, in der Luftröhre eingetrocknet, fest, da es ja nicht ausgespült wird, sondern allmählich hinzuströmt und darin festgehalten wird, und es ruft Husten hervor. Der Fluß, der in den Luftröhrchen stecken bleibt, weil sie nur enge Durchgänge haben, macht der Luft Beengung und das macht Atemnot, sobald es (dem Kranken) immer (an Luft) fehlt und er immer (solche) einatmen will.... Wenn die Krankheit feuchter wird, bekommen die Betreffenden Empyeme statt dieser Schwindsuchterscheinungen, wenn es trockener wird, anstelle von Empyemen Schwindsucht.

Leute mit Empyemen kann man an folgendem erkennen: zu Anfang befällt sie Schmerz in den Seiten; wenn der Eiter sich aber zusammengezogen hat, so sucht der Schmerz sie in gleicher Weise heim, Husten stellt sich ein, der Kranke wirft Eiter aus und bekommt Atembeschwerden. Falls (der Eiter) noch nicht durchgebrochen ist, so ruft Schütteln (des Kranken) in der Seite Plätschern wie in einem Schlauch hervor....

Die Brustfellentzündung soll man auf folgende Weise behandeln: man bringe das Fieber während 7 Tagen nicht zum Aufhören; als Getränk verwende man entweder Essighonig oder Essig und Wasser; hiervon soll man aber möglichst viel geben, damit eine Durch- feuchtung (der Lunge) erfolgt und diese durch ihr Auftreten Auswerfen bewirkt. Den Schmerz beruhige man durch erhitzende Mittel. Als Krankensuppe gebe man, was Auswurf bewirkt. Auch Bäder verwende am 4. Tage, am 5. und 6. Tage salbe man mit Öl. Am siebten Tag gebe man ein Bad, falls das Fieber nicht nachlassen will, damit durch das Bad der Schweißausbruch erfolge ..."

Im Kapitel 19 („Die Stellen am Menschen“) wird zur Therapie bei Schwindsucht vermerkt ([45], VIII, S. 67):

„Die an Schwindsucht (Erkrankten behandele man) im Übrigen ebenso (Anm: wie alle Patienten mit Brustfellentzündung), nur (gebe man) ihnen nicht viele Speisen zugleich und an der Zukost nicht mehr als an Speisen, ferner gebe man zum Essen gewässerten Wein, damit er nicht erhitze und dem Körper in seinem schwachen $\mathrm{Zu}$ stand Hitze bereite und nicht gleichzeitig beides (Wein und Zukost) heiß mache und Fieberhitze erzeuge.“

Im 2. Buch der Krankheiten, dessen Verfasser unbekannt sind, finden sich Hinweise zur Behandlung der Schwindsucht, geprägt von den sog. humoralpathologischen Vorstellungen der FlüsseTheorie. Im Kapitel 48 der „Behandlung der Lungenleiden ( = des 5. Flusses aus der Kopfhöhle: nach der Brusthöhle)“ wird mit der Überschrift „Eine andere Lungenkrankheit (=Schwindsucht)“ angeordnet [45], XVIII, S. 17):

„Im Endstadium keine Behandlung mehr einleiten. Im Anfangsstadium: Nieswurz und Magenreizmittel geben, dagegen keine Abführmittel, nur Klistiere geben. Gute Ernährung, Bewegung im Freien, Vorsicht vor Wind und Sonne.“

Zusammenfassend sind dem Corpus Hippocraticum im Zusammenhang mit der erwähnten Phthisis folgende Symptome zu entnehmen: Chronischer, häufig letal endender Krankheitsverlauf mit Anorexie, Gewichtsabnahme bis zur Kachexie und Kräfteverfall; anhaltender quälender Husten mit reichlich eitrigem, zum Teil übel riechendem Auswurf mit körniger Konsistenz und Hämoptysen; Fieber, Schüttelfrost und Schweißneigung; zunehmende Atemnot und Neigung zu Synkopen; thorakale Flankenschmerzen, Geräuschphänomene im Falle einer Pleuritis. Über die Epidemiologie ist dokumentiert: Es handelt sich um eine Erkrankung mit höchster Mortalität und Morbidität unter der damaligen Bevölkerung; häufiger Krankheitsbeginn im Winter, Maximum der Erkrankungen zwischen dem 18. und 35. Lebensjahr. Die Symptome und epidemiologischen Angaben sind mit den pathomorphologischen und klinischen Folgen der Lungentuberkulose und ihrer lokalen Komplikationen vereinbar.

\section{Diskussion}

Die Entwicklung des griechischen Alphabets und die Verbreitung eines einheitlichen Alphabets phönizischen Ursprungs werden auf das 8. -6. Jahrhundert v. Chr. datiert [46], so dass es nicht verwundert, dass aus der protogeometrischen (1025-900 v. Chr.) und geometrischen Zeit (900-700 v. Chr.) keine Textsammlungen, sondern lediglich mündliche Überlieferungen existieren. Insofern kann über das heilkundliche Wissen der frühgriechischen Epoche nur spekuliert werden. Ohne Zweifel wurden aber bereits Jahrhunderte vor Hippokrates Behandlungen mit dem Ziel 
durchgeführt, Krankheitssymptome und Verletzungsfolgen zu lindern. Dies lässt sich zum Beispiel aus den Überlieferungen der Asklepiaden um 800 v. Chr. und der ionischen Naturphilosophen aus dem 6. Jahrhundert v. Chr. ableiten. Die homerischen Epen und die Schriften Herodots vermitteln als älteste erhaltene Schriftquellen eine Konzentration auf Maßnahmen zur Therapie von Traumata, die im Rahmen von Kriegshandlungen entstanden, während Krankheiten mit chronischem Verlauf als nicht zu behandeln galten und gleichzeitig in Verbindung mit einer religiösen Schuldzuweisung gesellschaftlich stigmatisiert wurden. Aus diesen Quellen könnte gefolgert werden, dass ein Patient im Falle einer spezifischen Erkrankung, die mit einem chronisch progredienten Kräfteverfall einherging, nicht behandelt wurde und er nicht nur seine Funktion in der sozialen Gemeinschaft verlor, sondern geächtet wurde.

Letztlich ist jede Interpretation der Schriftquellen spekulativ, die sich auf eine nicht nachgewiesene Erkrankung bezieht; denn es ist völlig unbekannt, ob nicht gerade die Symptome der Tuberkulose - wie Husten, Gewichtsabnahme, Appetitlosigkeit, Atemnot etc. - im Einzelfall behandelt wurden, weil sie als Zeichen einer akuten Störung der Befindlichkeit, nicht als Ausdruck einer chronischen inneren Erkrankung gewertet wurden. Hinzu kommt, dass die heroisierenden Epen nicht zwingend die Situation der Heilkunde der frühgriechischen Gesellschaft reflektieren, da die Kriegshelden ihren Status naturgemäß in zunehmender Weise dann verlieren, wenn ihre „Kräfte dahinschwinden“, Frauen, Kinder und ältere Menschen diesem Status jedoch nicht unterliegen.

In den erhaltenen frühesten griechischen Quellen finden sich keine klinischen Hinweise, die den begründeten Verdacht auf die Existenz der Tuberkulose rechtfertigen. Auch die häufig zitierte Textstelle, in der Herodot die Erkrankung des Kriegsführers Pharnuches nach dem Sturz vom Pferd mit nachfolgender Hämoptoe und Kachexie beschreibt [47], kann nicht als Beweis einer sekundären Lungentuberkulose verstanden werden: Aus heutiger Sicht ist nach dem Sturz eine Rippen- (möglicherweise Serien-)Fraktur mit Verletzung des Lungenparenchyms wahrscheinlich, die mit einem Hämatopneumothorax und Hämoptysen einherging. Nach Quellenlage bleibt es allerdings unklar, ob es sich um eine Hämoptoe oder um eine Hämatemesis handelte. Denkbar wäre ebenfalls eine Verletzung im Bereich des NasenRachenraumes, die mit einer Blutung einherging. Es fehlt auch der Hinweis, ob dieses klinische Phänomen lediglich einmalig oder rezidivierend auftrat; im Falle einer progredienten Lungentuberkulose kann von wiederholten wenn nicht gar von persistierenden Hämoptysen ausgegangen werden. Als Ursache für den chronischen, über Jahre dauernden posttraumatischen Verlauf die Exazerbation einer alten Lungentuberkulose anzunehmen [48], ist vor dem Hintergrund der differenzialdiagnostischen Möglichkeiten ebenfalls spekulativ; in Betracht kommt eine progrediente respiratorische Insuffizienz primär und am wahrscheinlichsten durch chronisch pneumonische, unspezifische Infektionen auf dem Boden der Lungenparenchymverletzung, posttraumatische Bronchiektasenbildungen und/oder rezidivierende Lungenembolien.

Auch wenn sowohl in den homerischen Epen als auch in den vorhippokratischen Schriften und Dichtungen keine richtungsweisenden Hinweise auf die Tuberkulose zu finden sind, wird in die- sen Werken der Ursprung des Begriffes „Schwinden“ der Kräfte, „Siechen“ und „Siechtum“ deutlich. Bei Homer noch nicht zu finden, aber von Herodot mehrfach erwähnt ist der Begriff $\varphi \theta i ́ \sigma ı \varsigma$ (phthisis), der zunächst die Folgen einer Kriegsverletzung mit chronischem und letztendlich letalem Verlauf bezeichnet. Dieser aus den o.g. Gründen mit Makeln behaftete Krankheitsverlauf, verbunden mit dem langsamen, unaufhaltsamen Prozess des Sterbens, war mehrfach Thema schriftlicher Exkurse, zumal die verabscheuungswürdige und gleichzeitig jedoch tragische Komponente in der zeitgenössischen Auffassung von Heldentum besonders in der epischen Prosa interessierte.

Etymologisch entstand der Terminus $\varphi \theta i ́ \sigma ı$ (phthisis [49]) aus $\varphi \theta i ́ \mu \omega$ (phthimio; vernichten, hinschwinden), möglicherweise

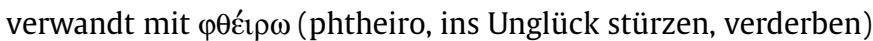
mit der Wurzel $\varphi \theta^{\prime}$ (phthi), wobei der Ursprung des Anlauts se-

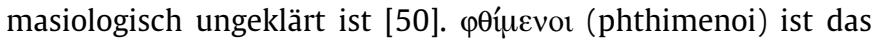
substantivierte Partizip im Plural und wird mit „die Dahinsiechenden“, die „siechenden Kräfte, die zum Tode führen“ und auch mit „die Toten, die Gestorbenen, die Vergangenen, die Entschwundenen, die Dahinsterbenden“ übersetzt [50]. Die intransitive Form des Terminus $\varphi \theta \dot{\mu} \mu \omega$ (phthimo) bedeutet „hinschwinden, umkommen, abhärmen, abnehmen, untergehen, vertilgen, viel zu leiden haben“ [51,52], die transitive Form ,jemanden töten, zugrunde richten, verderben, aufreiben, verzehren“[50].

Aus den Schriften des Corpus Hippocraticum lässt es sich nicht ableiten, dass unter $\varphi \theta i ́$ бı (phthisis) eine monokausale, ätiologisch definierte Erkrankung verstanden wurde. Angesichts der damaligen humoral-pathologischen Vorstellungen existierte kein Krankheitsbegriff, der der heutigen Definition entsprochen haben könnte. Dennoch bietet die hippokratische Terminologie eine semantische Differenzierung des Herodotschen Terminus $\varphi \theta i ́ \sigma ı \varsigma$ (phthisis), der nicht länger jedes chronische Siechtum bis zum Tode meinte [52], sondern primär die Symptomatik bronchopulmonaler und pleuraler Affektionen und deren Komplikationen mit langdauerndem und häufig letalem Verlauf beschrieb. Diese Differenzierung erlaubt in der Schriftenanalyse eine richtungsweisende klinische Einteilung, die auf eine Lungenerkrankung deutet. Vor dem Hintergrund der differenzialdiagnostischen Möglichkeiten handelt es sich aber dennoch lediglich um den ersten Schritt der klinischen und terminologischen Spezifizierung; die Differenzialdiagnosen umfassen unspezifische pulmonale und pleurale Infekte, Mykosen, Aspergillosen, parasitäre und neoplastische Erkrankungen, Ornithosen, Fibrosen, Sarkoidose, Histiozytosis X, rheumatoide granulomatöse Veränderungen und pulmonale sekundäre Manifestationen von Systemerkrankungen. Bezogen auf die Beobachtungen, die einen Krankheitsverlauf bis 9 Jahren notieren, wird das differenzialdiagnostische Spektrum enger: Malignome (z.B. kleinzellige und nichtkleinzellige Bronchialkarzinome) kommen dann in prognostischer Hinsicht [53] nicht mehr in Betracht. Da Pneumonien und parapneumonische Komplikationen unspezifischer Infektionen in der Regel mit deutlich erhöhten Temperaturen und weniger mit „gelindem Fieber“ [54] einhergehen, kann auch diese Differenzialdiagnose nicht in jedem Fall zutreffen. Allerdings erlaubt das Spektrum der angegebenen Temperaturerhöhungen unterschiedlichen Ausmaßes zahlreiche Interpretationsmöglichkeiten. Die Art des Auswurfes lässt aufgrund der als purulent und körnig beschriebenen Beschaffenheit an die käsige Sekretion ei- 
ner floriden Tuberkulose denken, schließt aber weitere Möglichkeiten nicht aus; ähnliche Formen des Auswurfes mit hoher Viskosität können bekanntlich bei Exsikkose - z. B. infolge von Fieberzuständen - auftreten, bei Aspergillosen, wenn mycelienhaltiges Sputum abgehustet wird, oder im Rahmen von Bronchiektasenbildungen beobachtet werden, die nach chronischem Verlauf auch mit der Bildung von Broncholithen einhergehen können. Die Tuberkulose gehört bekanntlich dabei lediglich zu einer Ursache von Bronchiektasien, die entzündlich und postentzündlich ebenfalls nach unspezifischen Infekten und posttraumatisch oder kongenital entstehen, mit dem Symptomentrias Husten, Auswurf und Atemnot einhergehen und bevorzugt im Kindesalter auftreten [55]. Dass die im Corpus Hippocraticum als Phthisis bezeichnete pulmonale Affektion vorwiegend zwischen dem 18 . und 35. Lebensjahr entsteht [54], spricht nicht zwingend gegen eine unspezifische Genese der Erkrankung.

Da die Genese dieser mit dem Terminus phthisis bezeichneten Lungenerkrankungen trotz der detaillierten und organbezogenen Notiz ausschließlich anhand der klinischen Symptomatik nicht zu klären ist und ohne mikrobiologische Diagnostik prinzipiell auch keine sichere Differenzierung zwischen spezifischen und unspezifischen Bakteriosen möglich ist [56], folgt hinsichtlich des dokumentierten Spektrums der Symptome, dass unter phthisis Lungenerkrankungen mit chronischem und häufig letalem Verlauf unterschiedlicher Genese subsummiert wurden. Die etymologischen Unsicherheiten und die differenzialdiagnostischen Möglichkeiten erlauben jedoch nicht die Schlussfolgerung, dass es die Tuberkulose in der hellenistischen Epoche der altgriechischen Historie nicht gab. Stattdessen spricht die Summe der Einzelbeobachtungen für die Existenz spezifischer Er- krankungen, auch wenn die hippokratischen Vorstellungen der sog. Humoralpathologie dabei zum Teil massiv die klinische Wahrnehmung in dem Versuch überlagern, die Ursachen, Folgen und den Verlauf von Krankheiten durch einen Säftefluss von Schleim, Blut, Galle und Gewebswasser zu erklären. Wenn z.B. von einem Schleim gesprochen wird, der in der Kopfhöhle verfault und dann Richtung Lunge fließt, um dort die Erkrankung auszulösen [54], ist dies nach heutigem Wissenstand eine nicht zutreffende Erklärung der Pathogenese pulmonaler Infektionen. Es kann hieraus jedoch nicht abgeleitet werden, dass die medizinischen Kenntnisse zu gering waren, die klinischen Befunde von den Erkrankungen Innerer Organe in exakter Weise zu erfassen. Die Dokumentationen im Corpus Hippocraticum beweisen das Gegenteil: Werden die Schriften auf die klinischen Hinweise reduziert analysiert, finden sich detaillierte Dokumentationen von Symptomen und demografischen Daten, die mit der Klinik tuberkulöser Erkrankungen vereinbar sind. Daher kann von der Existenz der Tuberkulose in der hellenistischen Epoche ausgegangen werden, ohne dass allerdings sichere Aussagen zur Epidemiologie der Erkrankung möglich sind.

Das Corpus Hippocraticum blieb über Jahrhunderte als medizinische Lehrbuchsammlung richtungsweisend für die Lehre und $\mathrm{Er}-$ kenntnisse über die Phthisis; weder in der griechischen, noch in der römischen Antike wurden wesentliche weiterführende Erkenntnisse gewonnen, die das Werk ergänzen konnten.

Aus technischen Grüinden kann das Literaturverzeichnis nicht abgedruckt werden. Es kann beim Verfasser angefordert werden. 